NOTA CIENTÍFICA

\title{
Extensión del rango de distribución de Asthenes sclateri (Aves: Fur- nariidae) en el Perú
}

1 Unión Ornitológica del Perú (UNOP), Investigadora Asociada National Museum of Natural History, Smithsonian Institution. Dirección postal: Av. San Luís 2773, San Borja, Lima 41, Perú. Email Grace Servat: grace.servat@gmail.com 2 DESCO (Centro de Estudios y Promoción del Desarrollo), Calle rénocion Umacollo, Arequipa, Perú. Email ca_reservasalinas@yahoo.com

3 Museo de Historia Natural de la Universidad Nacional de San Agustín, Arequipa, Perú.

Presentado: $29 / 10 / 2008$ Aceptado: $18 / 12 / 2008$

Publicado online: 26/02/2009

\section{Range distribution extension of Asthenes sclateri (Aves: Furnariidae) in Peru \\ Grace P. Servat ${ }^{1}$, Kenny Caballero ${ }^{2}$, José Luis Velásquez ${ }^{2}$, Renzo Alcocer ${ }^{3}$, Natali Hurtado ${ }^{2,3}$ y Maykol Rodríguez ${ }^{3}$}

\begin{abstract}
Resumen
Extendemos el rango de distribución en el Perú de Asthenes sclateri ("Canastero de Puna"), especie reportada hasta la fecha para el Departamento de Puno. Las observaciones de varios individuos de la especie (documentadas con fotos y especimenes) fueron hechas en pajonales altoandinos aledaños a bosques de Polylepis en el Departamento de Arequipa.
\end{abstract}

Palabras clave: Asthenes sclateri, distribución, Arequipa, pajonal altoandino, bosque de Polylepis.

\section{Abstract}

We extent the distribution range in Peru of Asthenes sclateri, Cabanis ("Puna Canastero"), a species reported only for the Puno Department. The observations of many individuals of the species (documented with pictures and specimens) were made in high Andean pastures nearby Polylepis woodlands in the Arequipa Department.

Keywords: Asthenes sclateri, distribution, Arequipa, high Andean pastures, Polylepis woodlands.
El 4 de mayo del año 2007, durante un censo de aves realizado en el remanente de bosque de queñoa (Polylepis rugulosa, Rosaceae, Pilger) en el área denominada "Cabrerías" (Departamento y Provincia de Arequipa, laderas del volcán Chachani, $71^{\circ} 29^{\prime} \mathrm{W} ; 16^{\circ} 16^{\prime} \mathrm{S}, 3820 \mathrm{~m}$ de altitud) (Fig. 1), se tuvo el primer avistamiento de un individuo del género Asthenes el cual estaba alimentándose de artrópodos en el suelo pedregoso de la vegetación circundante constituida principalmente por pastos y pequeños arbustos. El individuo presentaba estriaciones de color negro en la región dorsal que contrastaban con la coloración rojiza-marrón de la espalda y los márgenes externos de la cola rojizos (Fig. 2). En días sucesivos (5-7 de mayo) pudimos observar algunos individuos más en la zona de estudio (Servat

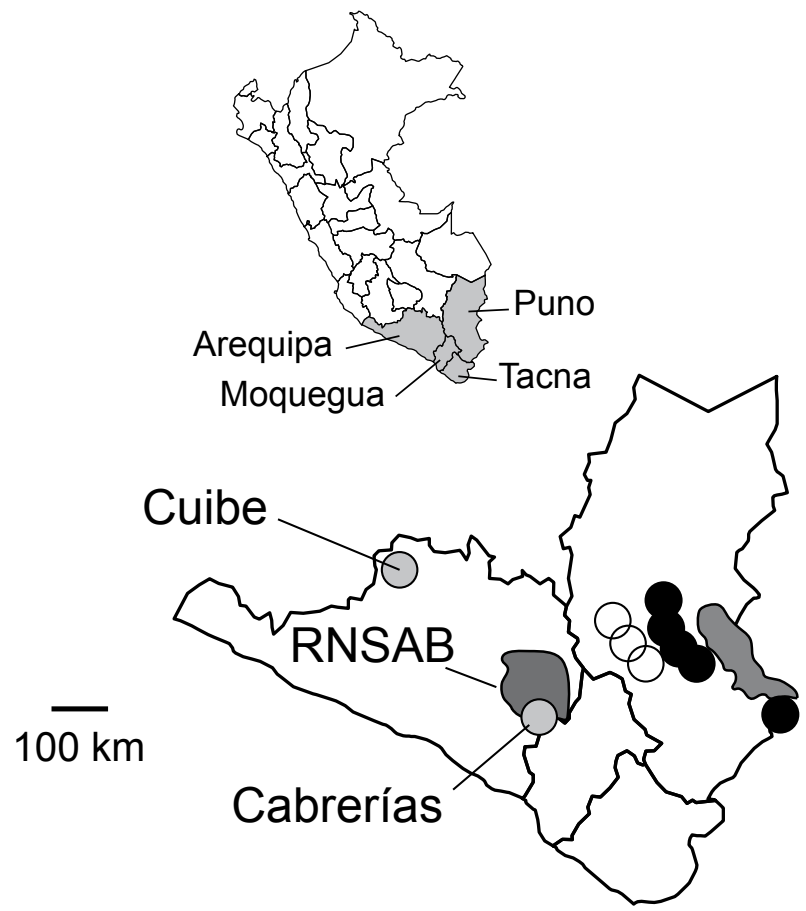

Figura 1. Distribución de Asthenes sclateri. Los círculos negros corresponden a registros confirmados para el Dpto. de Puno, los círculos blancos son registros por confirmar (Schulenberg et. al 2007). Los círculos grises representan las localidades de Cuibe y Cabrerías esta ultima en la RNSAB (Ver texto). 2007a) y capturamos, mediante el uso de redes de neblina, un individuo hembra (GSV 1123, Tabla 1) el cual fue preparado como ejemplar de estudio para su posterior identificación. En visitas posteriores a la misma localidad realizadas el 24 de noviembre de 2007 y el 9 de mayo de 2008 se capturaron dos individuos mas, una hembra (GSV 1151, Tabla 1) y un macho (MUSA 1902, Tabla 1), respectivamente. El 26 de marzo de 2008 un cuarto espécimen (MUSA 1863, Tabla 1) colectado por R. Alcocer en el distrito de Cotahuasi (Provincia de La Unión) expande la distribución norte de la especie. Todos los especimenes mencionados, se encuentran depositados en la Colección Ornitológica del Museo de la Universidad de San Agustín de Arequipa (MUSA).

Una revisión posterior de la literatura y la comparación de especímenes con la colección ornitológica del Carnegie Museum en los Estados Unidos, nos confirma que los especímenes colectados en Cabrerías corresponden a Asthenes sclateri ("Canastero de Puna”), especie reportada hasta entonces únicamente para el Departamento de Puno en Perú (Fig. 1). La coloración del plumaje y las medidas promedio de longitud del pico, ala, tarso, cola y total de los especimenes colectados en Arequipa tuvieron mayor similitud con los especimenes de $A$. ("punensis") sclateri que con los de A. wyatti (Tabla 1).

La especie $A$. sclateri fue descrita por Cabanis (1878) y permaneciendo vigente hasta que Cory y Hellmayr (1925) la consideraron como un sinónimo de $A$. hudsoni. En el mismo tratado se reconocieron como especies válidas a $A$. wyatti, $A$. punensis, y $A$. anthoides. Posteriormente, Fjeldså y Krabbe en el libro "Birds of the High Andes" (1990) reconocen nuevamente como especie a $A$. sclateri y la separan de $A$. wyatti, $A$. punensis, y $A$. anthoides, durante ese mismo ańo Sibley y Monroe (1990) en el libro "Distribution and Taxonomy of Birds of the World" consideran a $A$. sclateri como un sinónimo de $A$. punensis, sin embargo la especie $A$. sclateri tendría precedencia sobre $A$. punensis como lo indica el último tratamiento del grupo (Remsen 2003, Remsen et. al. 2007) el cual es el que se sigue en la presente publicación. 


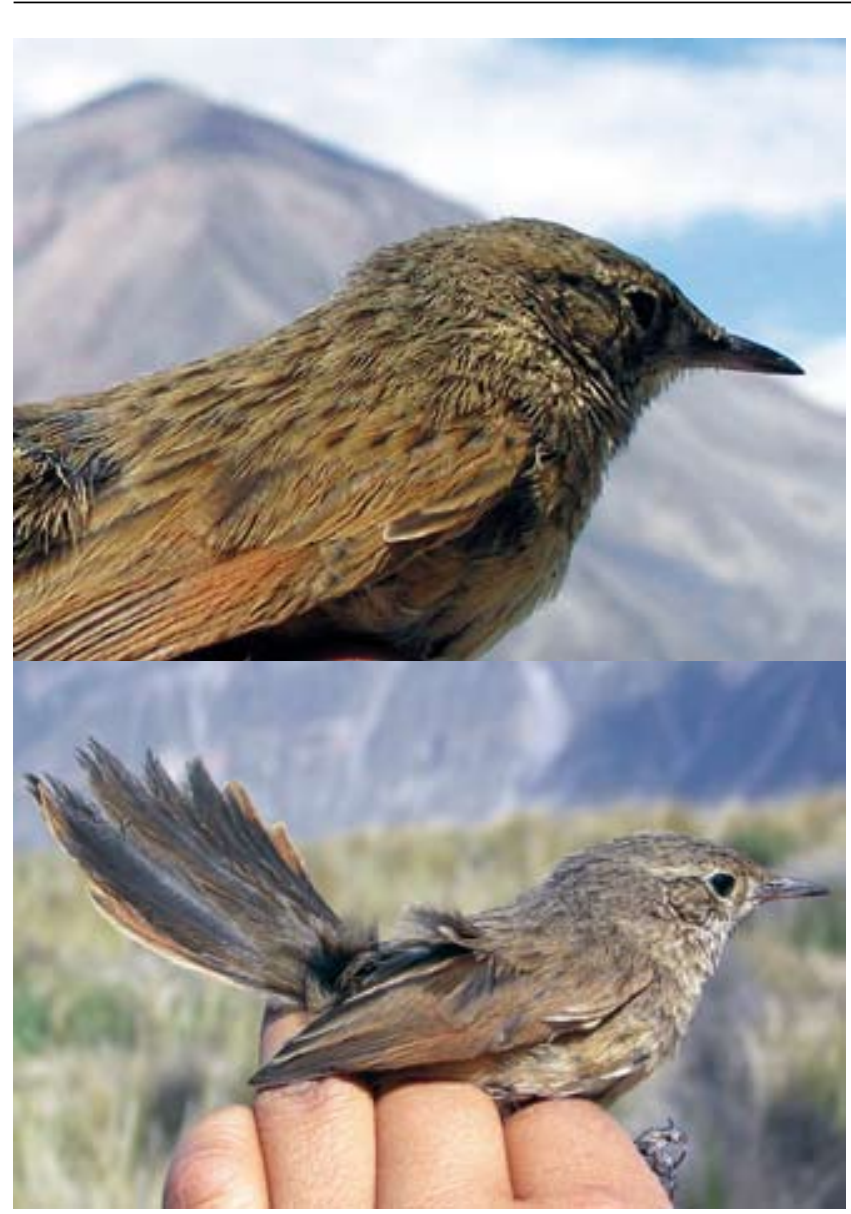

Figura 2. Asthenes sclateri, especie caracterizada por las estriaciones dorsales (foto superior), y bordes externos de las rectrices de color rojizo (foto inferior). Nótese el hábitat (pajonal de puna seca) en el fondo (Fotos G. Servat, Cabrerías).

\section{Agradecimientos}

Queremos agradecer a E. López Tejada curador de la colección ornitológicas del Museo de la Universidad de San Agustín, Arequipa (MUSA), a Bradley C. Livezey y Stephen P. Rogers curadores del Carnegie Museum of Natural History (CMNH) en Philadelphia por permitirnos revisar las colecciones a su cargo. Asimismo, agradecemos a J. Fjeldså y T. Schulenberg por su ayuda con la identificación de la especie. La realización de este trabajo no hubiera sido posible sin el apoyo de H. Zeballos, J. Ochoa y todas las personas del Contrato de Administración
Parcial de Operaciones de la Reserva Nacional Salinas y Aguada Blanca (DESCO, Arequipa). Así también ha sido invaluable la ayuda en el trabajo de campo y gabinete de G. Ordinola, $\mathrm{H}$. Zamora y J. P. Ludeńa.

\section{Literatura Citada}

Cabanis J.L. 1878. Journal fur Ornithologie 26, Pp.196.

Cory C.B. \& C.E. Hellmayr. 1925. Catalogue of Birds of the Americas Part IV Furnariidae-Dendrocolaptidae. Field Museum of Natural History Publications. Zoological Series, Chicago.

Fjeldså J. \& N. Krabbe. 1990. Birds of the high Andes. Copenhagen. Zool. Mus., University of Copenhagen and Svendborg, Apollo Books. 876 p.

IUCN. (En linea). 2008 IUCN Red List of Threatened Species. $<$ http: $\| w w w . i u c n r e d l i s t . o r g ~>$. Acceso 12/10/2008.

Meyer De Schauensee R. 1970. A Guide to the Birds of South America. Livingston Publ., Wynnewood, PA.

Remsen J.V. 2003. Family Furnariidae (Ovenbirds). In: J. del Hoyo, A. Elliott \& D.A. Christie, eds. Handbook of the birds of the world. Lynx Edicions, Barcelona, Spain. Pp. 162-357.

Remsen J.V., Jr., C.D. Cadena, A. Jaramillo, M. Nores, J.F. Pacheco, et al. 2007. (En linea). A classification of the bird species of South America, Part 6: Suboscine Passeriformes (Furnariidae). American Ornithologists'Union. <www. museum.lsu.edu/ Remsen/SACCBaseline.html $>$. Acceso $12 / 10 / 2008$.

Schulenberg T., D.F. Stotz, D.F. Lane, J.P. O’Neill, \& T.A. Parker III. 2007. Birds of Peru. Princeton Field Guides.

Servat G.P. 2007a. Riqueza, Diversidad, Abundancia y Densidad de las poblaciones de aves asociadas a bosques de Polylepis rugulosa (ROSACEAE) en la Reserva Nacional de Salinas y Aguada Blanca. Reporte DESCO - junio 2007.

Sibley C.G. \& B.L. Monroe, Jr. 1990. Distribution and taxonomy of birds of the world. Yale University Press, New Haven.

Tabla 1. Peso y medidas promedio de los especimenes examinados en las colecciones ornitológicas del CMNH y MUSA. ( $\left.{ }^{1}\right)$ la especie denominada "punensis" corresponde a A. sclateri (ver texto). ${ }^{2}$ ) Los números de catalogo indican la fecha y localidad de los especimenes: 1) CMNH 71076, 3/7/1913, Ecuador: Mountain Chimborazo, 13,000 ft; 2) CMNH 45201, 2/4/1914, Colombia: Sierra Nevada de Santa Marta, Cerro de Caracas. 10000 - 12000 ft; 3) CMNH 45202, 2/4/1914, (localidad = 2); 4) CMNH 71077, 4/7/1913, Ecuador: Mountain Chimborazo. 13000 ft; 5) CMNH 81222, 20/4/1920, Bolivia: Cochabamba, Caluya. 3500 m; 6) CMNH 81221, 20/4/1920, (localidad = 5); 7) CMNH 119681, 21/8/1921, Bolivia: Cochabamba, Colomi. 3500 m; 8) CMNH 125051, 30/1/1938, Bolivia: Cochabamba, Cuchicanchi. 3085 m; 9) CMNH 85454, 22/8/1921, Bolivia: Cochabamba, Colomi. 3500 m; 10) CMNH 125049, 29/1/1938, Bolivia: Cochabamba, Cerro San Benito; 11) GSV 1123, 5/5/2007, Perú: Departamento y Provincia de Arequipa, Distrito de Cayma, Bosque de Polylepis rugulosa de Cabrerías, laderas del volcán Chachani, 71 ${ }^{\circ} 29^{\prime} \mathrm{W} ; 16^{\circ} 16^{\prime}$ 'S, 3835 m; 12) GSV 1151, 24/11/2007 (localidad= 11); 13) MUSA 1863, 26/3/2008, Perú: Departamento Arequipa, Provincia de La Unión, Distrito de Cotahuasi, Cuibe, ca. 3500 m; 14) MUSA 1902, 9/5/2008 (localidad= 11).

\begin{tabular}{|c|c|c|c|c|c|c|c|c|c|}
\hline \multirow{2}{*}{ Especie $^{1}$} & \multirow{2}{*}{ Sexo } & \multirow{2}{*}{$\begin{array}{c}\text { N.o de } \\
\text { ejemplares }\end{array}$} & \multirow{2}{*}{ N.o de catalogo ${ }^{2}$} & \multicolumn{5}{|c|}{ Longitud (mm) } & \multirow{2}{*}{$\begin{array}{c}\text { Peso } \\
\text { (g) }\end{array}$} \\
\hline & & & & Pico & Ala & Tarso & Cola & Total & \\
\hline A. wyatti & $\mathrm{H}$ & 3 & CMNH 71076, 45201, 45202 & 11,2 & 48,0 & 21,8 & 66,7 & 129,0 & ND \\
\hline A. wyatti & M & 1 & CMNH 71077 & 11,2 & 60,0 & 26,0 & 81,0 & 148,0 & ND \\
\hline A. ("punensis") sclateri & $\mathrm{H}$ & 4 & CMNH $81222,81221,119681,125051$ & 9,8 & 58,2 & 24,2 & 92,5 & 159,0 & ND \\
\hline A. ("punensis") sclateri & M & 2 & CMNH 85454,125049 & 10,9 & 58,5 & 22,9 & 105,0 & 163,0 & ND \\
\hline A. sclateri & $\mathrm{H}$ & 3 & GSV 1123, 1151, MUSA 1863 & 12,0 & 64,0 & 25,2 & 87,0 & 163,0 & 22 \\
\hline A. sclateri & M & 1 & MUSA 1902 & 12,7 & 66,0 & 25,1 & 76,0 & 154,0 & 21 \\
\hline
\end{tabular}

